\title{
3D hydrodynamical model stellar atmospheres of metal-poor red giants
}

\author{
Remo Collet ${ }^{1}$, Martin Asplund ${ }^{2}$ and Regner Trampedach ${ }^{2}$ \\ ${ }^{1}$ Department of Astronomy \& Space Physics, BOX 515, SE-751 20 Uppsala, Sweden \\ email: remo@astro.uu.se \\ ${ }^{2}$ Research School of Astronomy \& Astrophysics, Cotter Road, Weston ACT 2611, Australia
}

\begin{abstract}
We investigate the main differences between static 1D and 3D time-dependent model stellar atmospheres of red giants at very low metallicities. We focus in particular on the impact of 3D LTE spectral line formation on the derivation of elemental abundances for the extremely metal-poor $([\mathrm{Fe} / \mathrm{H}] \approx-5.3)$ red giant $\mathrm{HE} 0107-5240$.
\end{abstract}

Keywords. Stars: late-type, stars: atmospheres, methods: numerical, hydrodynamics, line: formation, stars: abundances

\section{Introduction}

Recently 3D time-dependent hydrodynamical simulations of convection in the atmosphere of late-type stars (Asplund et al. 1999) have indicated that the deviations of the $3 \mathrm{D}$ structure from the 1D stratification can produce significant differences in the strength of synthetic spectral lines and ultimately affect the determination of stellar abundances. Here we present preliminary estimates of the effects of convection in 3D model stellar atmospheres of extremely metal-poor red giants $([\mathrm{Fe} / \mathrm{H}] \leqslant-3)$ on the derivation of chemical abundances.

\section{Calculations}

We use the 3D radiative-hydrodynamics code by Stein \& Nordlund (1998) to simulate convection in the atmosphere of a red giant star with effective temperature $\mathrm{T}_{\text {eff }}=5130$ $\mathrm{K}$, surface gravity $\log \mathrm{g}=2.2$ and metallicity $[\mathrm{Fe} / \mathrm{H}]=-3$. We perform spectral line formation calculations in local thermodynamic equilibrium (LTE) for several elements using our 3D model atmosphere and a 1D MARCS model atmosphere (Asplund et al. 1997) generated for the same stellar parameters and a micro-turbulence $\xi=2.0 \mathrm{~km} \mathrm{~s}^{-1}$ (figure 1, left). While both 3D and $1 \mathrm{D}$ models are constructed for a metallicity $[\mathrm{Fe} / \mathrm{H}]=-3$, for the line formation calculations we assume the chemical composition to be essentially the one of the extremely metal-poor red giant HE 0107-5240 (Christlieb et al. 2002). As the 1D MARCS stratification of our model atmosphere at $[\mathrm{Fe} / \mathrm{H}]=-3$ closely resembles the one of HE 0107-5240, the differential 3D-1D abundance analysis should also provide an estimate of the $3 \mathrm{D}$ effects for this extremely metal-poor star.

\section{Results}

As previously found by Asplund et al. (1999) for dwarfs and subgiants 3D model atmospheres of metal-poor red giants also appear to be significantly cooler at the surface than their 1D counterparts. Consequently, at a given abundance, the populations of neutral atoms and molecules in these upper layers are enhanced in 3D models comparing 
Table 1. HE 0107-5240: 3D-1D abundance corrections (dex). For neutral and singly ionised elements we refer to the line list by Christlieb et al. (2004). Corrections for C, N and O are estimated using fiducial $\mathrm{CH}, \mathrm{NH}$ and $\mathrm{OH}$ molecular lines.

\begin{tabular}{ccccccccc}
\hline Li I & Na I & Mg I & Al I & Si I & Ca I & Ca II & Sc II & Ti II \\
\hline-0.4 & -0.25 & -0.15 & -0.4 & -0.15 & -0.4 & -0.05 & -0.05 & -0.05 \\
\hline Cr I & Mn I & Fe I & Fe II & Co I & Ni I & CH & NH & OH \\
\hline-0.7 & -0.3 & -0.4 & +0.07 & -0.4 & -0.5 & -0.7 & -0.8 & -0.6 \\
\hline
\end{tabular}
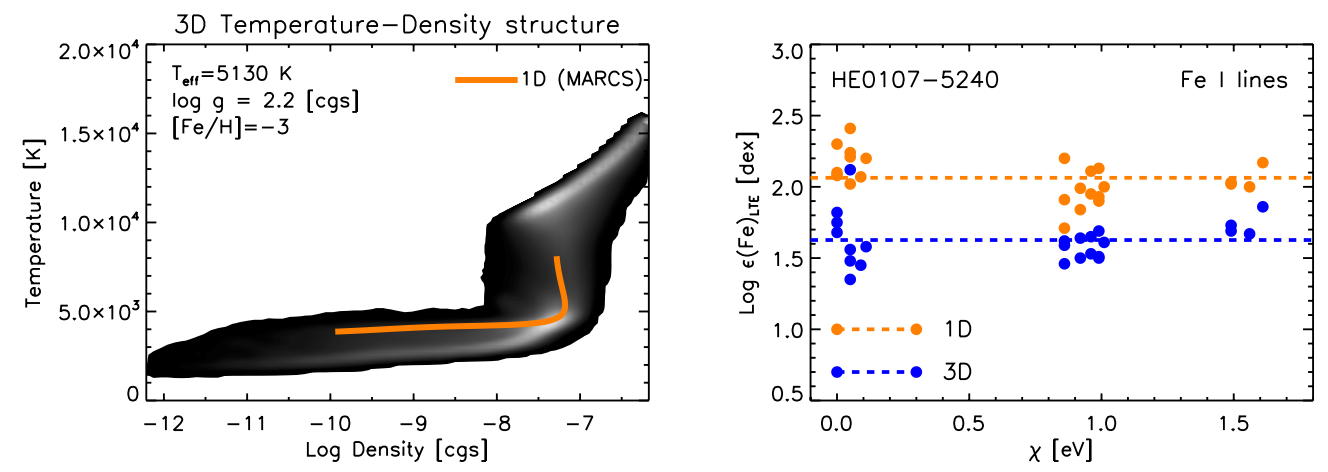

Figure 1. Left: Temperature-density distribution in our 3D model red giant atmosphere. and corresponding 1D MARCS stratification. Right: HE 0107-5240's iron abundance from Fe I lines as a function of excitation potential of lower levels for both 1D and 3D analysis.

to 1D models. Lines of these species generally form in the upper atmospheric layers and appear stronger in $3 \mathrm{D}$ models than in $1 \mathrm{D}$, leading to negative 3D-1D differential abundance corrections (table 1). Further investigations are however needed to study possible departures from LTE and equilibrium chemistry.

\section{Conclusions}

We performed simulations of convection in the atmosphere of very metal-poor red giants. We then studied 3D LTE spectral line formation at $[\mathrm{Fe} / \mathrm{H}] \approx-5$ and derived estimates of the 3D-1D abundance corrections to be applied to the classical analysis of the extremely metal-poor star HE 0107-5240. We found these corrections to be significant and negative when lines from neutral atoms and from molecules were used as indicators. In particular we found a 3D-1D abundance correction of -0.4 dex for Fe I (figure 1, right) and of about -0.7 dex for $\mathrm{C}, \mathrm{N}$ and $\mathrm{O}$ (as estimated from molecular lines).

\section{References}

Asplund, M., Gustafsson, B., Kiselman, D., \& Eriksson, K. 1997, A\&A 318, 521

Asplund, M., Nordlund, Å., Trampedach, R., \& Stein, R.F. 1999, A\&A (Letters) 346, L17

Christlieb, N., Bessel, M.S., Beers, T.C., Gustafsson, B., Korn, A., Barklem, P.S., Karlsson, T., Mizuno-Wiedner, M., \& Rossi S. 2002, Nature, 419, 904

Christlieb, N., Gustafsson, B., Korn, A.J., Barklem, P.S., Beers, T.C., \& Bessel, M.S. 2004, ApJ, 603,708

Stein, R.F. \& Nordlund, Å 1998, ApJ 499, 914 